\title{
A NEW PINCHING THEOREM FOR CLOSED HYPERSURFACES WITH CONSTANT MEAN CURVATURE IN $S^{n+1 *}$
}

\author{
HONG-WEI $\mathrm{XU}^{\dagger}$ AND LING TIAN ${ }^{\dagger}$
}

\begin{abstract}
We investigate the generalized Chern conjecture, and prove that if $M$ is a closed hypersurface in $S^{n+1}$ with constant scalar curvature and constant mean curvature, then there exists an explicit positive constant $C(n)$ depending only on $n$ such that if $|H|\langle C(n)$ and $S\rangle \beta(n, H)$, then $S>\beta(n, H)+\frac{3 n}{7}$, where $\beta(n, H)=n+\frac{n^{3} H^{2}}{2(n-1)}+\frac{n(n-2)}{2(n-1)} \sqrt{n^{2} H^{4}+4(n-1) H^{2}}$.
\end{abstract}

Key words. Closed hypersurface, Pinching phenomenon, Mean curvature, Scalar curvature, Second fundamental form.

AMS subject classifications. 53C40, 53C42.

1. Introduction. Let $M^{n}$ be an $n$-dimensional closed hypersurface in the unit sphere $S^{n+1}$ with constant scalar curvature and constant mean curvature. Denote by $H$ and $S$ the mean curvature and the squared norm of the second fundamental form of $M$, respectively. It follows from the Gauss equation that the scalar curvature of $M$ is given by $R=n(n-1)+n^{2} H^{2}-S$.

A famous rigidity theorem due to Simons [15], Lawson [10] and Chern-do CarmoKobayashi [8] says that if $M$ is a closed minimal hypersurface in $S^{n+1}$ satisfying $S \leq n$, then $S \equiv 0$ and $M$ is the great sphere $S^{n}$, or $S \equiv n$ and $M$ is the Clifford torus $S^{k}\left(\sqrt{\frac{k}{n}}\right) \times S^{n-k}\left(\sqrt{\frac{n-k}{n}}\right)$. Afterward, Li-Li [11] improved Simons' pinching constant for $n$-dimensional closed minimal submanifolds in $S^{n+p}$ to $\max \left\{\frac{n}{2-1 / p}, \frac{2}{3} n\right\}$. Peng-Terng [13] proved that there exists a positive constant $\delta(n)$ depending only on $n$ such that if $n \leq S \leq n+\delta(n)$, then $S=n$. Later Yang-Cheng [25] improved the pinching constant $\delta(n)$ to $\frac{n}{3}$. In 1993, S. P. Chang [2, 7] solved Chern's conjecture in dimension 3 . For closed minimal hypersurfaces in $S^{n+1}$, the scalar curvature pinching phenomenon without the assumption of constant scalar curvature was also investigated by several authors $[5,9,14,17,23]$ etc.

More generally, we would like to propose the following

The Generalized Chern Conjecture. For closed hypersurfaces in the unit sphere $S^{n+1}$ with constant scalar curvature and constant mean curvature, the values $S$ of the squared norm of the second fundamental forms must be discrete.

Set $\alpha(n, H)=n+\frac{n^{3}}{2(n-1)} H^{2}-\frac{n(n-2)}{2(n-1)} \sqrt{n^{2} H^{4}+4(n-1) H^{2}}$. In 1990, the first author [18] proved the generalized Simons-Lawson-Chern-do Carmo-Kobayashi theorem for compact submanifolds with parallel mean curvature in a sphere.

THEOREM A. Let $M$ be an $n$-dimensional oriented compact submanifold with parallel mean curvature in an $(n+p)$-dimensional unit sphere $S^{n+p}$. If $S \leq C_{1}(n, p, H)$, then $M$ is either a totally umbilic sphere $S^{n}\left(\frac{1}{\sqrt{1+H^{2}}}\right)$, a Clifford hypersurface in an

\footnotetext{
*Received February 25, 2010; accepted for publication February 1, 2011. Research supported by the NSFC, Grant No. 11071211, 10771187; the Trans-Century Training Programme Foundation for Talents by the Ministry of Education of China; and the Natural Science Foundation of Zhejiang Province, Grant No. 101037.

$\dagger$ Center of Mathematical Sciences, Zhejiang University, Hangzhou 310027, China (\{xuhw; tian ling\}@cms.zju.edu.cn).
} 
$(n+1)$-sphere, or the Veronese surface in $S^{4}\left(\frac{1}{\sqrt{1+H^{2}}}\right)$. Here the constant $C_{1}(n, p, H)$ is defined by

$$
C_{1}(n, p, H)= \begin{cases}\alpha(n, H), & p=1, \text { or } p=2 \text { and } H \neq 0 \\ \frac{n}{2-\frac{1}{p}}, & p \geq 2 \text { and } H=0, \\ \min \left\{\alpha(n, H), \frac{n+n H^{2}}{2-\frac{1}{p-1}}+n H^{2}\right\}, & p \geq 3 \text { and } H \neq 0 .\end{cases}
$$

Consequently, we have the following

Corollary A (also see $[1,6]$ ). Let $M$ be an n-dimensional closed hypersurface with constant mean curvature $(H \neq 0)$ in $S^{n+1}$. If $S \leq \alpha(n, H)$, then $M$ is either a totally umbilical sphere $S^{n}\left(\frac{1}{\sqrt{1+H^{2}}}\right)$, or a Clifford hypersurface $S^{n-1}\left(\frac{1}{\sqrt{1+\lambda^{2}}}\right) \times$ $S^{1}\left(\frac{\lambda}{\sqrt{1+\lambda^{2}}}\right)$. Here $\lambda=\frac{1}{2(n-1)}\left[n|H|+\sqrt{n^{2} H^{2}+4(n-1)}\right]$.

In [19], H. W. Xu improved the pinching constant $C_{1}(n, p, H)$ in Theorem A to

$$
C_{2}(n, p, H)= \begin{cases}\alpha(n, H), & p=1, \text { or } p=2 \text { and } H \neq 0, \\ \min \left\{\alpha(n, H), \frac{1}{3}\left(2 n+5 n H^{2}\right)\right\}, & \text { otherwise. }\end{cases}
$$

In [3], S. P. Chang showed that any closed hypersurface in $S^{4}$ with constant scalar curvature and constant mean curvature must be an isoparametric hypersurface. Recently, Suh and Yang [16] improved Yang and Cheng's pinching theorem for closed minimal hypersurfaces, and proved the following

Theorem B. Let $M^{n}(n \geq 4)$ be an $n$-dimensional closed minimal hypersurface with constant scalar curvature in $S^{n+1}$. If $S>n$, then $S>n+\frac{3}{7} n$.

In this paper, we extend the result of Suh and Yang to the case where $M$ is a closed hypersurface with constant scalar curvature and small constant mean curvature. Putting $\beta(n, H)=n+\frac{n^{3} H^{2}}{2(n-1)}+\frac{n(n-2)}{2(n-1)} \sqrt{n^{2} H^{4}+4(n-1) H^{2}}$, we prove the following

Main TheORem. Let $M^{n}(n \geq 4)$ be an $n$-dimensional closed hypersurface in $S^{n+1}$ with constant scalar curvature and constant mean curvature. Then there exists an explicit positive constant $C(n)$ depending only on $n$, such that if $|H|<C(n)$ and $S>\beta(n, H)$, then $S>\beta(n, H)+\frac{3 n}{7}$.

We have the following corollary immediately.

Corollary. Let $M^{n}(n \geq 4)$ be an n-dimensional closed hypersurface in $S^{n+1}$ with constant scalar curvature and constant mean curvature $(H \neq 0)$. If $|H|<C(n)$ and $\beta(n, H) \leq S \leq \beta(n, H)+\frac{3 n}{7}$, then $S=\beta(n, H)$ and $M$ is an isoparametric hypersurface $S^{1}\left(\frac{1}{\sqrt{1+\mu^{2}}}\right) \times S^{n-1}\left(\frac{\mu}{\sqrt{1+\mu^{2}}}\right)$, where $\mu=\frac{1}{2}\left[n|H|+\sqrt{n^{2} H^{2}+4(n-1)}\right]$.

The following problem seems very interesting.

Open Problem. For an n-dimensional closed hypersurface $M$ in $S^{n+1}$ with constant scalar curvature $R$ and constant mean curvature $H(H \neq 0)$, set $\mu_{k}=$ $\frac{n|H|+\sqrt{n^{2} H^{2}+4 k(n-k)}}{2 k}$. Suppose that $\alpha(n, H) \leq S \leq \beta(n, H)$. Can one prove that $M$ must be the isoparametric hypersurface $S^{k}\left(\frac{1}{\sqrt{1+\mu_{k}^{2}}}\right) \times S^{n-k}\left(\frac{\mu_{k}}{\sqrt{1+\mu_{k}^{2}}}\right), k=1,2, \cdots, n-$ 1 ?

It should be mentioned that the scalar curvature pinching phenomenon for closed constant mean curvature hypersurfaces in $S^{n+1}$ has been investigated in $[4,21,22]$ etc. 
2. Fundamental formulas. Throughout this paper let $M^{n}$ be an $n$-dimensional closed hypersurface in $S^{n+1}$ with constant scalar curvature and constant mean curvature. We shall make use of the following convention on the range of indices:

$$
1 \leq A, B, C, \cdots \leq n+1, \quad 1 \leq i, j, k, \cdots \leq n .
$$

Choose a local orthonormal frame field $\left\{e_{A}\right\}$ in $S^{n+1}$ such that, restricted to $M,\left\{e_{i}\right\}$ are tangent to $M$. Let $\left\{\omega_{A}\right\}$ and $\left\{\omega_{A B}\right\}$ be the dual frame field and the connection 1-forms of $S^{n+1}$, respectively. Restricting these forms to $M$, we have

$$
\omega_{n+1}=0, \quad \omega_{n+1 i}=\sum_{j=1}^{n} h_{i j} \omega_{j}, \quad h_{i j}=h_{j i} .
$$

The second fundamental form of $M$ can be written as $h=\sum_{i, j=1}^{n} h_{i j} \omega_{i} \otimes \omega_{j} \otimes e_{n+1}$. Denote by $H$ and $S$ the mean curvature of $M$ and the squared norm of $h$, respectively. Then

$$
S=\sum_{i, j=1}^{n} h_{i j}^{2}, \quad H=\frac{1}{n} \sum_{i=1}^{n} h_{i i} .
$$

For an arbitrary fixed point $p \in M$, choose $\left\{e_{A}\right\}$ such that

$$
H \geq 0, \quad h_{i j}=\lambda_{i} \delta_{i j}, \quad S=\sum_{i=1}^{n} \lambda_{i}^{2} .
$$

Set

$$
\begin{gathered}
f_{k}:=\sum_{i} \lambda_{i}^{k}, \\
h_{i j k}:=\nabla_{k} h_{i j}, h_{i j k l}:=\nabla_{l} \nabla_{k} h_{i j}, h_{i j k l s}:=\nabla_{s} \nabla_{l} \nabla_{k} h_{i j} .
\end{gathered}
$$

The Gauss equation, Codazzi equation and Ricci formula can be written as

$$
\begin{gathered}
R_{i j k l}=\delta_{i k} \delta_{j l}-\delta_{i l} \delta_{j k}+h_{i k} h_{j l}-h_{i l} h_{j k}, \\
h_{i j k}=h_{i k j}, \\
h_{i j k l}-h_{i j l k}=\sum_{m}\left(h_{m j} R_{m i k l}+h_{i m} R_{m j k l}\right), \\
h_{i j k l s}-h_{i j k s l}=\sum_{m}\left(h_{m j k} R_{m i l s}+h_{i m k} R_{m j l s}+h_{i j m} R_{m k l s}\right),
\end{gathered}
$$

where $R_{i j k l}$ is the curvature tensor of $M$. So the scalar curvature of $M$ is given by $R=n(n-1)+n^{2} H^{2}-S$.

When $H$ and $S$ are constants, by a similar computation as in [13], we get

$$
\sum_{i, j, k} h_{i j k}^{2}=S(S-n)+n^{2} H^{2}-n H f_{3},
$$




$$
\sum_{k} h_{i j k k}=(n-S) h_{i j}+\left(\sum_{m} h_{i m} h_{m j}-\delta_{i j}\right) n H,
$$

$$
h_{i j i j}-h_{j i j i}=\left(\lambda_{i}-\lambda_{j}\right)\left(1+\lambda_{i} \lambda_{j}\right)
$$

Following [20], we have

$$
\begin{aligned}
\frac{1}{3} \triangle f_{3} & =(n-S) f_{3}+n H\left(f_{4}-S\right)+2 \sum_{i, j, k} \lambda_{i} h_{i j k}^{2}, \\
\frac{1}{4} \triangle f_{4} & =(n-S) f_{4}+n H\left(f_{5}-f_{3}\right)+(2 A+B), \\
\frac{1}{6} \triangle f_{3}^{2}= & (n-S) f_{3}^{2}+n H f_{3}\left(f_{4}-S\right)+2 f_{3} \sum_{i, j, k} \lambda_{i} h_{i j k}^{2} \\
& +3 \sum_{j}\left(\sum_{i} \lambda_{i}^{2} h_{i i j}\right)^{2},
\end{aligned}
$$

where

$$
A=\sum_{i, j, k} \lambda_{i}^{2} h_{i j k}^{2}, \quad B=\sum_{i, j, k} \lambda_{i} \lambda_{j} h_{i j k}^{2} .
$$

By (2.1), (2.4) and the Laplacian of $\sum h_{i j k}^{2}$, we obtain the following

$$
\begin{aligned}
\sum_{i, j, k, l} h_{i j k l}^{2}= & (S-2 n-3)\left(S^{2}-n S+n^{2} H^{2}-n H f_{3}\right) \\
& -\frac{3 n H}{2}\left[(n-S) f_{3}-n S H+n H f_{4}\right] \\
& +3(A-2 B)-6 n H \sum_{i, j, k} \lambda_{i} h_{i j k}^{2} .
\end{aligned}
$$

On the other hand, it follows from (2.3) that

$$
\begin{aligned}
\sum_{i, j, k, l} h_{i j k l}^{2} & \geq \sum_{i, j, k, l} u_{i j k l}^{2}+\frac{3}{4} \sum_{i \neq j}\left(h_{i j i j}-h_{j i j i}\right)^{2} \\
& =\sum_{i, j, k, l} u_{i j k l}^{2}+\frac{3}{2}\left(S f_{4}-f_{3}^{2}-2 S^{2}+n S-n^{2} H^{2}+2 n H f_{3}\right)
\end{aligned}
$$

where

$$
u_{i j k l}:=\frac{1}{4}\left(h_{i j k l}+h_{j k l i}+h_{k l i j}+h_{l i j k}\right) .
$$


3. Several lemmas. To prove our main result, we need several lemmas. From now on, we take $\tilde{S}=S-n H^{2}, \sum_{i, j, k} h_{i j k}^{2}=t \tilde{S}^{2}$. Because of $(2.1)$, it is easy to see that

$$
t=\frac{\tilde{S}-n}{\tilde{S}}+\frac{n H\left[H \tilde{S}-\left(f_{3}-H S\right)\right]}{\tilde{S}^{2}} .
$$

Put

$$
\begin{aligned}
f & =\sum_{i}\left(\lambda_{i}^{2}-\frac{f_{3}-H S}{\tilde{S}} \lambda_{i}-\frac{S^{2}-n H f_{3}}{n \tilde{S}}\right)^{2} \\
& =f_{4}-\frac{\left(f_{3}-H S\right)^{2}}{\tilde{S}}-\frac{S^{2}}{n},
\end{aligned}
$$

and $\tilde{\lambda}_{i}=\lambda_{i}-H, i=1, \cdots, n$. At any point $p \in M$, let $\lambda_{1}=\max _{i}\left\{\lambda_{i}\right\}, \lambda_{2}=\min _{i}\left\{\lambda_{i}\right\}$.

LEMMA 1. Let $M^{n}$ be an $n$-dimensional closed hypersurface in $S^{n+1}$ with constant scalar curvature and constant mean curvature. Then at any point $p \in M$, we have

$$
\begin{aligned}
& f \geq \frac{\left(\tilde{\lambda}_{1}-\tilde{\lambda}_{2}\right)^{2}}{\tilde{\lambda}_{1}^{2}+\tilde{\lambda}_{2}^{2}}\left(\tilde{\lambda}_{1} \tilde{\lambda}_{2}+\frac{\tilde{S}}{n}\right)^{2}, \\
& A-B \leq \frac{1-\alpha}{3}\left(\tilde{\lambda}_{1}-\tilde{\lambda}_{2}\right)^{2} t \tilde{S}^{2}, \\
& \sum_{j}\left(\sum_{i} \lambda_{i}^{2} h_{i i j}\right)^{2} \leq \frac{1+2 \alpha}{3} t \tilde{S}^{2} f,
\end{aligned}
$$

$$
\left(\sum_{i, j, k} \lambda_{i} h_{i j k}^{2}\right)^{2} \leq\left\{\frac{1}{3}(A+2 B)-\frac{4}{3} \sum_{k} \frac{(n+2)\left(\sum_{i} \lambda_{i}^{2} h_{i i k}\right)^{2}}{(n+2) \tilde{S}+2 n \tilde{\lambda}_{k}^{2}}\right\} t \tilde{S}^{2}
$$

where $\alpha \sum_{i, j, k} h_{i j k}^{2}=\sum_{i} h_{i i i}^{2}$ and $\alpha$ satisfies $0 \leq \alpha \leq 1$.

Proof. (3.3), (3.4) and (3.5) can be proved by using the same method as in [24]. We only need to prove (3.6). Consider equations

$$
\left\{\begin{array}{l}
\sum_{i, j} P_{i j k} h_{i j}=0 \\
\sum_{i, j} P_{i j k} \delta_{i j}=0
\end{array}\right.
$$

where $P_{i j k}:=\left(\lambda_{i}+\lambda_{j}+\lambda_{k}\right) h_{i j k}-\left(h_{i j} y^{k}+h_{i k} y^{j}+h_{j k} y^{i}\right)-\left(\delta_{i j} x^{k}+\delta_{i k} x^{j}+\delta_{j k} x^{i}\right)$. This together with the fact that $H$ and $S$ are constants implies

$$
\left\{\begin{array}{l}
2 \sum_{i} \lambda_{i}^{2} h_{i i k}-\left(2 \lambda_{k}^{2}+S\right) y^{k}-\left(2 \lambda_{k}+n H\right) x^{k}=0 \\
\left(2 \lambda_{k}+n H\right) y^{k}+(2+n) x^{k}=0 .
\end{array}\right.
$$

We then have the following solution.

$$
x^{k}=-\frac{2\left(2 \lambda_{k}+n H\right) \sum_{i} \lambda_{i}^{2} h_{i i k}}{2 n\left(\lambda_{k}-H\right)^{2}+(n+2) \tilde{S}}, y^{k}=\frac{2(n+2) \sum_{i} \lambda_{i}^{2} h_{i i k}}{2 n\left(\lambda_{k}-H\right)^{2}+(n+2) \tilde{S}}, k=1, \cdots, n .
$$


By a similar argument as in [24], we get

$$
\begin{aligned}
& \left(\sum_{i, j, k} \lambda_{i} h_{i j k}^{2}\right)^{2}=\frac{1}{9}\left(\sum_{i, j, k} P_{i j k} h_{i j k}\right)^{2} \\
\leq & \frac{1}{9} \sum_{i, j, k} P_{i j k}^{2} \sum_{i, j, k} h_{i j k}^{2} \\
= & \left\{\frac{1}{3}(A+2 B)-\frac{4}{3} \sum_{k} \frac{(n+2)\left(\sum_{i} \lambda_{i}^{2} h_{i i k}\right)^{2}}{(n+2) \tilde{S}+2 n \tilde{\lambda}_{k}^{2}}\right\} t \tilde{S}^{2} .
\end{aligned}
$$

LEMma 2. Let $M^{n}$ be an n-dimensional closed hypersurface in $S^{n+1}$ with constant scalar curvature and constant mean curvature. Then

$$
f \leq \frac{\left(\tilde{\lambda}_{1}-\tilde{\lambda}_{2}\right)^{2}}{4} \tilde{S}-\frac{\tilde{S}^{2}}{n}
$$

Proof. Put $\tilde{f}_{3}=\sum_{i} \tilde{\lambda}_{i}^{3}, \tilde{f}_{4}=\sum_{i} \tilde{\lambda}_{i}^{4}$. It follows from (3.2) that

$$
f=\sum_{i}\left(\tilde{\lambda}_{i}^{2}-\frac{\tilde{f}_{3}}{\tilde{S}} \tilde{\lambda}_{i}-\frac{\tilde{S}}{n}\right)^{2}=\tilde{f}_{4}-\frac{\tilde{f}_{3}^{2}}{\tilde{S}}-\frac{\tilde{S}^{2}}{n} .
$$

By the definitions of $\lambda_{1}$ and $\lambda_{2}$, we have

$$
\sum_{i} \tilde{\lambda}_{i}^{2}\left(\tilde{\lambda}_{i}-\tilde{\lambda}_{1}\right)\left(\tilde{\lambda}_{i}-\tilde{\lambda}_{2}\right) \leq 0
$$

Then

$$
\tilde{f}_{4} \leq\left(\tilde{\lambda}_{1}+\tilde{\lambda}_{2}\right) \tilde{f}_{3}-\tilde{\lambda}_{1} \tilde{\lambda}_{2} \tilde{S}
$$

Thus

$$
\begin{aligned}
f & \leq\left(\tilde{\lambda}_{1}+\tilde{\lambda}_{2}\right) \tilde{f}_{3}-\frac{\tilde{f}_{3}^{2}}{\tilde{S}}-\tilde{\lambda}_{1} \tilde{\lambda}_{2} \tilde{S}-\frac{\tilde{S}^{2}}{n} \\
& \leq \frac{\left(\tilde{\lambda}_{1}+\tilde{\lambda}_{2}\right)^{2}}{4} \tilde{S}-\tilde{\lambda}_{1} \tilde{\lambda}_{2} \tilde{S}_{-}-\frac{\tilde{S}^{2}}{n}=\frac{\left(\tilde{\lambda}_{1}-\tilde{\lambda}_{2}\right)^{2}}{4} \tilde{S}-\frac{\tilde{S}^{2}}{n} .
\end{aligned}
$$

Lemma 3. Let $M^{n}$ be an $n$-dimensional closed hypersurface in $S^{n+1}$ with constant scalar curvature and constant mean curvature. Then

$$
\int_{M^{n}}(A-2 B) d v=\int_{M^{n}}\left(\tilde{S} f+\frac{t \tilde{S}^{3}}{n}+g_{1}\right) d v
$$

where $g_{1}=H^{2}\left(n f+t \tilde{S}^{2}\right)-H t \tilde{S}\left(f_{3}-H S\right)$. 
Proof. Note that

$$
\begin{aligned}
& \sum_{i, j, k, l, m} h_{l m i k} h_{i j} h_{j m} h_{l k} \\
= & \sum_{i, j, k, l, m}\left(h_{l m i} h_{i j} h_{j m} h_{l k}\right)_{k}-\sum_{i, j, k, l, m} h_{l m i} h_{i j k} h_{j m} h_{l k} \\
& -\sum_{i, j, k, l, m} h_{l m i} h_{i j} h_{j m k} h_{l k} \\
= & \frac{1}{3} \sum_{k, l}\left(\left(f_{3}\right)_{l} h_{l k}\right)_{k}-2 B .
\end{aligned}
$$

Since $H$ and $S$ are constants, we have

$$
\begin{aligned}
& \sum_{i, j, k, l, m} h_{l m i k} h_{i j} h_{j m} h_{l k} \\
= & \sum_{i, j, k, l, m}\left(h_{l m k i}+\sum_{r} h_{r m} R_{r l i k}+\sum_{r} h_{l r} R_{r m i k}\right) h_{i j} h_{j m} h_{l k} \\
= & \sum_{i, j, k, l, m}\left(h_{l m k} h_{i j} h_{j m} h_{l k}\right)_{i}-\sum_{i, j, k, l, m} h_{l m k} h_{i j i} h_{j m} h_{l k} \\
& -\sum_{i, j, k, l, m} h_{l m k} h_{i j} h_{j m i} h_{l k}-\sum_{i, j, k, l, m} h_{l m k} h_{i j} h_{j m} h_{l k i} \\
& +n H f_{3}+S f_{4}-S^{2}-f_{3}^{2} \\
= & -A+n H f_{3}+S f_{4}-S^{2}-f_{3}^{2} .
\end{aligned}
$$

Putting $X=\sum_{k}\left(\sum_{l}\left(f_{3}\right)_{l} h_{l k}\right) e_{k}$, we get $\operatorname{div}(X)=\sum_{k, l}\left(\left(f_{3}\right)_{l} h_{l k}\right)_{k}$. By (3.9), (3.10) and the divergence theorem, we obtain

$$
\int_{M^{n}}(A-2 B) d v=\int_{M^{n}}\left(S f_{4}-f_{3}^{2}+n H f_{3}-S^{2}\right) d v .
$$

This together with (3.1) and the definitions of $f$ and $\tilde{S}$ implies (3.8).

4. Curvature estimates. The crucial point of the proof of Main Theorem is to give a proper estimate of the RHS of (2.8).

We begin with an estimate of $\sum_{i, j, k, l} u_{i j k l}^{2}$. Define

$$
f_{i j}=\sum_{k} h_{i k} h_{k j}-\frac{f_{3}-H S}{\tilde{S}} h_{i j}-\frac{S^{2}-n H f_{3}}{n \tilde{S}} \delta_{i j}, \quad i, j=1, \cdots, n,
$$

which satisfy

$$
\sum_{i, j} h_{i j} f_{i j}=0, \quad \sum_{i, j} \delta_{i j} f_{i j}=0, \quad \sum_{i, j} f_{i j}^{2}=f .
$$

Putting

$$
F:=-A+\frac{1}{2} S f+\frac{f_{3}-H S}{\tilde{S}}\left(\sum_{i, j, k} \lambda_{i} h_{i j k}^{2}-t \tilde{S}^{2} H\right)+\frac{t \tilde{S}^{2} S}{n},
$$


we have

$$
\sum_{i, j, k, l} u_{i j k l} h_{i j} f_{k l}=F
$$

$$
\sum_{i, j, k} u_{i j k l} h_{i j k}=-\frac{3}{2} \sum_{i} \lambda_{i}^{2} h_{i i l} \lambda_{l}-\frac{3 n H}{2} \sum_{i} \lambda_{i}^{2} h_{i i l}=-\frac{1}{2}\left(f_{3}\right)_{l}\left(\lambda_{l}+n H\right),
$$

$$
\sum_{i, j, k, l} u_{i j k l} h_{i j} \delta_{k l}=-\frac{1}{2} t \tilde{S}^{2}, \quad \sum_{i, j, k} h_{i j k} f_{i j} h_{k l}=\sum_{i} \lambda_{i}^{2} h_{i i l} \lambda_{l}=\frac{1}{3}\left(f_{3}\right)_{l} \lambda_{l} .
$$

Set

$$
\begin{aligned}
U_{i j k l}:= & u_{i j k l}-\alpha_{1}\left(h_{i j} f_{k l}+f_{i j} h_{k l}\right)+\left(\beta_{i} h_{j k l}+\beta_{j} h_{i k l}+\beta_{k} h_{l i j}+\beta_{l} h_{i j k}\right) \\
& +\gamma h_{i j} h_{k l}+\delta\left(h_{i j} \delta_{k l}+\delta_{i j} h_{k l}\right)+\zeta \delta_{i j} \delta_{k l} .
\end{aligned}
$$

For any $\alpha_{1}, \beta_{i}, \gamma, \delta, \zeta \in \mathbf{R}, \sum_{i, j, k, l} U_{i j k l}^{2} \geq 0$. Choose

$$
\begin{aligned}
\alpha_{1} & =\frac{1}{S f-\frac{1}{18 t \tilde{S}^{2}} \sum_{j}\left(f_{3}\right)_{j}^{2} \lambda_{j}^{2}}\left[F+\frac{1}{12 t \tilde{S}^{2}} \sum_{j}\left(f_{3}\right)_{j}^{2}\left(\lambda_{j}^{2}+n H \lambda_{j}\right)\right], \\
\beta_{i} & =\frac{1}{8 t \tilde{S}^{2}}\left(f_{3}\right)_{i}\left(\frac{2 \alpha_{1}+3}{3} \lambda_{i}+n H\right), \\
\gamma & =-t H+\frac{\sum_{i, j, k} \lambda_{i} h_{i j k}^{2}}{\tilde{S}^{2}} \\
\delta & =-\frac{\sum_{i, j, k} \lambda_{i} h_{i j k}^{2}}{\tilde{S}^{2}} H+\frac{t\left(S+n H^{2}\right)}{2 n}, \\
\zeta & =\frac{\sum_{i, j, k} \lambda_{i} h_{i j k}^{2}}{\tilde{S}^{2}} H^{2}-\frac{t S}{n} H,
\end{aligned}
$$

respectively. By the symmetry of $U_{i j k l}=U_{k l i j}, U_{i j k l}=U_{j i k l}$ and (4.2)-(4.5), substituting these values into the inequality $\sum_{i, j, k, l} U_{i j k l}^{2} \geq 0$, we obtain

$$
\begin{aligned}
\sum_{i, j, k, l} u_{i j k l}^{2} \geq & \frac{1}{2 S f-\frac{1}{9 t \tilde{S}^{2}} \sum_{j}\left(f_{3}\right)_{j}^{2} \lambda_{j}^{2}}\left[2 F+\frac{1}{6 t \tilde{S}^{2}} \sum_{j}\left(f_{3}\right)_{j}^{2}\left(\lambda_{j}^{2}+n H \lambda_{j}\right)\right]^{2} \\
& +\frac{1}{4 t \tilde{S}^{2}} \sum_{j}\left(f_{3}\right)_{j}^{2}\left(\lambda_{j}+n H\right)^{2} \\
& +\frac{1}{\tilde{S}^{2}}\left(\sum_{i, j, k} \lambda_{i} h_{i j k}^{2}-t H \tilde{S}^{2}\right)^{2}+\frac{t^{2} \tilde{S}^{3}}{2 n}
\end{aligned}
$$


Noting the definition of $F$, we have

$$
\begin{aligned}
& \sum_{i, j, k, l} u_{i j k l}^{2} \geq \frac{1}{2\left[S f-\frac{1}{18 t \tilde{S}^{2}} \sum_{j}\left(f_{3}\right)_{j}^{2} \lambda_{j}^{2}\right]}\left\{\left[S f-\frac{1}{18 t \tilde{S}^{2}} \sum_{j}\left(f_{3}\right)_{j}^{2} \lambda_{j}^{2}\right]\right. \\
& +\left[-2 A+\frac{2\left(f_{3}-H S\right)}{\tilde{S}}\left(\sum_{i, j, k} \lambda_{i} h_{i j k}^{2}-t H \tilde{S}^{2}\right)+\frac{2 t \tilde{S}^{2} S}{n}\right. \\
& \left.\left.+\frac{2}{9 t \tilde{S}^{2}} \sum_{j}\left(f_{3}\right)_{j}^{2} \lambda_{j}^{2}+\frac{n H}{6 t \tilde{S}^{2}} \sum_{j}\left(f_{3}\right)_{j}^{2} \lambda_{j}\right]\right\}^{2} \\
& +\frac{1}{4 t \tilde{S}^{2}} \sum_{j}\left(f_{3}\right)_{j}^{2}\left(\lambda_{j}+n H\right)^{2} \\
& +\frac{1}{\tilde{S}^{2}}\left(\sum_{i, j, k} \lambda_{i} h_{i j k}^{2}-t H \tilde{S}^{2}\right)^{2}+\frac{t^{2} \tilde{S}^{3}}{2 n} \\
& \geq-2 A+\frac{1}{2} S f+\frac{1}{\tilde{S}^{2}}\left(\sum_{i, j, k} \lambda_{i} h_{i j k}^{2}-t H \tilde{S}^{2}\right)^{2} \\
& +\frac{2\left(f_{3}-H S\right)}{\tilde{S}}\left(\sum_{i, j, k} \lambda_{i} h_{i j k}^{2}-t H \tilde{S}^{2}\right)+\frac{t \tilde{S}^{2}}{n}\left(2 S+\frac{t \tilde{S}}{2}\right) \\
& +\frac{1}{36 t \tilde{S}^{2}} \sum_{j}\left(f_{3}\right)_{j}^{2}\left[7 \lambda_{j}^{2}+9\left(\lambda_{j}+n H\right)^{2}+6 n H \lambda_{j}\right] \\
& =-2 A+\frac{1}{2} S f+\frac{1}{\tilde{S}^{2}}\left(\sum_{i, j, k} \lambda_{i} h_{i j k}^{2}-t H \tilde{S}^{2}\right)^{2} \\
& +\frac{2\left(f_{3}-H S\right)}{\tilde{S}}\left(\sum_{i, j, k} \lambda_{i} h_{i j k}^{2}-t H \tilde{S}^{2}\right)+\frac{t \tilde{S}^{2}}{n}\left(2 S+\frac{t \tilde{S}}{2}\right) \\
& +\frac{1}{36 t \tilde{S}^{2}} \sum_{j}\left(f_{3}\right)_{j}^{2}\left(4 \lambda_{j}+3 n H\right)^{2} \text {. }
\end{aligned}
$$

It follows from $(2.7),(2.8),(4.6)$ and $S=\tilde{S}+n H^{2}$ that

$$
\begin{aligned}
& \frac{t \tilde{S}^{2}}{2}\left\{5 \tilde{S}-7 n-6-\frac{(4+3 n) t \tilde{S}}{n}-\frac{4 \tilde{S}}{n}\right\}+(5 A-6 B) \\
\geq & 2 \tilde{S} f+\frac{1}{\tilde{S}^{2}}\left(\sum_{i, j, k} \lambda_{i} h_{i j k}^{2}\right)^{2}+\frac{2\left(f_{3}-H S\right)}{\tilde{S}} \sum_{i, j, k} \lambda_{i} h_{i j k}^{2} \\
& +\frac{1}{36 t \tilde{S}^{2}} \sum_{j}\left(f_{3}\right)_{j}^{2}\left(4 \lambda_{j}+3 n H\right)^{2}+\tilde{g}_{2},
\end{aligned}
$$

where

$$
\begin{aligned}
\tilde{g}_{2}= & \left((6 n-2 t) \sum_{i, j, k} \lambda_{i} h_{i j k}^{2}-2 t \tilde{S}\left(f_{3}-H S\right)\right) H \\
& +\left(2 n f+\frac{3 n^{2} f}{2}+t^{2} \tilde{S}^{2}-\frac{5 n-4}{2} t \tilde{S}^{2}\right) H^{2}
\end{aligned}
$$


From (3.1) and (4.7), we have

$$
-6=-\frac{6 n}{n}=-\frac{6}{n}\left\{(1-t) \tilde{S}+\frac{n H\left(H \tilde{S}-\left(f_{3}-H S\right)\right)}{\tilde{S}}\right\}
$$

and

$$
\begin{aligned}
& t \tilde{S}^{2}\left\{\tilde{S}-2 n-(5-t) \frac{\tilde{S}}{n}\right\}+(5 A-6 B)-2 \tilde{S} f \\
\geq & \frac{1}{\tilde{S}^{2}}\left(\sum_{i, j, k} \lambda_{i} h_{i j k}^{2}\right)^{2}+\frac{2\left(f_{3}-H S\right)}{\tilde{S}} \sum_{i, j, k} \lambda_{i} h_{i j k}^{2} \\
& +\frac{1}{36 t \tilde{S}^{2}} \sum_{j}\left(f_{3}\right)_{j}^{2}\left(4 \lambda_{j}+3 n H\right)^{2}+g_{2},
\end{aligned}
$$

where

$$
\begin{aligned}
g_{2}= & \left(2(3 n-t) \sum_{i, j, k} \lambda_{i} h_{i j k}^{2}-\frac{10+3 n}{2} t \tilde{S}\left(f_{3}-H S\right)\right) H \\
& +\left(\frac{4+3 n}{2} n f+(5-n+t) t \tilde{S}^{2}\right) H^{2}
\end{aligned}
$$

Now we estimate the right hand side of (4.8). We first consider the following integral equality.

$$
\begin{aligned}
0= & \int_{M^{n}}\left\{\frac{1}{4} \triangle f_{4}-\frac{1+c_{0}}{6 \tilde{S}} \triangle f_{3}^{2}+\frac{\left(1+c_{0}\right)\left(1+c_{2}\right) H}{3} \triangle f_{3}\right\} \\
& -\int_{M^{n}} c_{1}\left\{(A-2 B)-\left(\tilde{S} f+\frac{t \tilde{S}^{3}}{n}+g_{1}\right)\right\} .
\end{aligned}
$$

Using (2.4), (2.5), (2.6) and (3.8), we see that there is a point $p_{0} \in M$ such that at $p_{0}$, for any $c_{0} \geq 0$ and $u>0$,

$$
\begin{aligned}
& \left(1-c_{1}\right) u \frac{t \tilde{S}^{3}}{n}-\left(2-c_{1}\right) u A-\left(1+2 c_{1}\right) u B+\left(t-c_{1}\right) u \tilde{S} f \\
= & -\frac{2 u\left(c_{0}+1\right)}{\tilde{S}}\left(f_{3}-H S\right) \sum_{i, j, k} \lambda_{i} h_{i j k}^{2}+c_{0} u t\left(f_{3}-H S\right)^{2} \\
& -\frac{u\left(c_{0}+1\right)}{3 \tilde{S}} \sum_{j}\left(f_{3}\right)_{j}^{2}+g_{3},
\end{aligned}
$$

where

$$
\begin{aligned}
g_{3}= & -\frac{u H}{\tilde{S}^{2}}\left\{\tilde{S}^{2} H\left[\left(\tilde{S}+n H^{2}\right)^{2}-t \tilde{S}^{2}\left(c_{1}-1\right)\right]+n H \tilde{S} f \xi\right. \\
& +\tilde{S}\left[-\tilde{S}\left[-2\left(\tilde{S}+n H^{2}\right)+t(\tilde{S}+\xi)\right]+\left(2+c_{0}\right) n f\right]\left(f_{3}-H S\right) \\
& -n H \tilde{S}\left(f_{3}-H S\right)^{2}+2 \tilde{S}\left(1+c_{0}\right)\left(n H^{2}-c_{2} \tilde{S}\right) \sum_{i, j, k} \lambda_{i} h_{i j k}^{2} \\
& \left.-n\left(\tilde{S}^{2} f_{5}-\left(f_{3}-H S\right)^{3}\right)\right\}, \\
\xi= & n H^{2}\left(1+c_{0}\right)-\tilde{S}\left(c_{1}+\left(1+c_{0}\right) c_{2}\right) .
\end{aligned}
$$


Adding (4.9) to (4.8), we obtain

$$
\begin{aligned}
& t \tilde{S}^{2}\left\{\tilde{S}-2 n-\left[5-t-\left(1-c_{1}\right) u\right] \frac{\tilde{S}}{n}\right\}+\left[5-\left(2-c_{1}\right) u\right] A \\
& -\left[6+\left(1+2 c_{1}\right) u\right] B-\left[2-\left(t-c_{1}\right) u\right] \tilde{S} f \\
\geq & \frac{1}{\tilde{S}^{2}}\left(\sum_{i, j, k} \lambda_{i} h_{i j k}^{2}\right)^{2}+\frac{2\left(f_{3}-H S\right)}{\tilde{S}} \sum_{i, j, k} \lambda_{i} h_{i j k}^{2}\left[1-\left(c_{0}+1\right) u\right]+c_{0} u t\left(f_{3}-H S\right)^{2} \\
& +\sum_{j}\left(f_{3}\right)_{j}^{2}\left\{\frac{\left(4 \lambda_{j}+3 n H\right)^{2}}{36 t \tilde{S}^{2}}-\frac{u\left(c_{0}+1\right)}{3 \tilde{S}}\right\}+g_{2}+g_{3} \\
= & \frac{1}{\tilde{S}^{2}}\left(\sum_{i, j, k} \lambda_{i} h_{i j k}^{2}\right)^{2}\left\{1-\frac{\left[1-\left(c_{0}+1\right) u\right]^{2}}{c_{0} u t}\right\} \\
& +c_{0} u t\left\{\left(f_{3}-H S\right)+\frac{1-\left(c_{0}+1\right) u}{c_{0} u t \tilde{S}} \sum_{i, j, k} \lambda_{i} h_{i j k}^{2}\right\}^{2} \\
& +\sum_{j}\left(f_{3}\right)_{j}^{2}\left\{\frac{\left(4 \lambda_{j}+3 n H\right)^{2}}{36 t \tilde{S}^{2}}-\frac{u\left(c_{0}+1\right)}{3 \tilde{S}}\right\}+g_{2}+g_{3} \\
\geq & \frac{1}{\tilde{S}^{2}}\left(\sum_{i, j, k} \lambda_{i} h_{i j k}^{2}\right)^{2}\left\{1-\frac{\left[u\left(c_{0}+1\right)-1\right]^{2}}{c_{0} u t}\right\} \\
& +\sum_{j}\left(f_{3}\right)_{j}^{2}\left\{\frac{\left(4 \lambda_{j}+3 n H\right)^{2}}{36 t \tilde{S}^{2}}-\frac{u\left(c_{0}+1\right)}{3 \tilde{S}}\right\}+g_{2}+g_{3} .
\end{aligned}
$$

Setting $\beta=\frac{\left\{\left(c_{0}+1\right) u-1\right\}^{2}}{c_{0} u}-t$, we choose $c_{0}$, $u$ such that $\beta \geq 0$. By (3.6), the inequality above becomes

$$
\begin{aligned}
& t \tilde{S}^{2}\left\{\tilde{S}-2 n-\left[5-t-\left(1-c_{1}\right) u\right] \frac{\tilde{S}}{n}\right\}+\left[5-\left(2-c_{1}\right) u\right] A \\
& -\left[6+\left(1+2 c_{1}\right) u\right] B-\left[2-\left(t-c_{1}\right) u\right] \tilde{S} f \\
\geq & -\frac{\beta}{t \tilde{S}^{2}}\left(\sum_{i, j, k} \lambda_{i} h_{i j k}^{2}\right)^{2}+\sum_{j}\left(f_{3}\right)_{j}^{2}\left\{\frac{\left(4 \lambda_{j}+3 n H\right)^{2}}{36 t \tilde{S}^{2}}-\frac{u\left(c_{0}+1\right)}{3 \tilde{S}}\right\}+g_{2}+g_{3} \\
\geq & -\beta\left\{\frac{1}{3}(A+2 B)-\frac{4}{27} \sum_{j} \frac{(n+2)\left(f_{3}\right)_{j}^{2}}{(n+2) \tilde{S}^{2}+2 n \tilde{\lambda}_{j}^{2}}\right\} \\
& +\sum_{j}\left(f_{3}\right)_{j}^{2}\left\{\frac{\left(4 \lambda_{j}+3 n H\right)^{2}}{36 t \tilde{S}^{2}}-\frac{u\left(c_{0}+1\right)}{3 \tilde{S}}\right\}+g_{2}+g_{3} .
\end{aligned}
$$


Hence we have

$$
\begin{aligned}
& t \tilde{S}^{2}\left\{\tilde{S}-2 n-\left[5-t-\left(1-c_{1}\right) u\right] \frac{\tilde{S}}{n}\right\}+\left\{5-\left(2-c_{1}\right) u+\frac{\beta}{3}\right\} A \\
& -\left\{6+\left(1+2 c_{1}\right) u-\frac{2 \beta}{3}\right\} B-\left[2-\left(t-c_{1}\right) u\right] \tilde{S} f \\
\geq & \sum_{j}\left(f_{3}\right)_{j}^{2}\left\{\frac{4}{27} \cdot \frac{(n+2) \beta}{(n+2) \tilde{S}+2 n \tilde{\lambda}_{j}^{2}}+\frac{\left(4 \lambda_{j}+3 n H\right)^{2}}{36 t \tilde{S}^{2}}-\frac{u\left(c_{0}+1\right)}{3 \tilde{S}}\right\} \\
& +g_{2}+g_{3} \\
= & \sum_{j}\left(f_{3}\right)_{j}^{2}\left\{\frac{4}{27} \cdot \frac{(n+2) \beta}{(n+2) \tilde{S}+2 n \tilde{\lambda}_{j}^{2}}+\frac{4 \tilde{\lambda}_{j}^{2}}{9 t \tilde{S}^{2}}-\frac{u\left(c_{0}+1\right)}{3 \tilde{S}}\right\} \\
& +g_{2}+g_{4} \\
\geq & \frac{1}{9 \tilde{S}} \sum_{j}\left(f_{3}\right)_{j}^{2}\left\{4 \sqrt{\frac{2(n+2) \beta}{3 n t}}-\frac{2(n+2)}{n t}-3 u\left(c_{0}+1\right)\right\}+g_{2}+g_{4},
\end{aligned}
$$

where

$$
g_{4}=g_{3}+\frac{4+3 n}{9 t \tilde{S}^{2}} \sum_{j}\left(f_{3}\right)_{j}^{2}\left(2 \lambda_{j}+\left(\frac{3}{4} n-1\right) H\right) H .
$$

5. Proof of the main theorem. Making use of the lemmas and estimates above, we shall prove our main theorem. We first show $t \geq \frac{1}{4}$. Suppose that $t \in\left[\frac{1}{9}, \frac{1}{4}\right)$. From (4.10) and $\frac{(n+2) \beta}{(n+2) \tilde{S}+2 n \tilde{\lambda}_{j}^{2}} \geq \frac{\beta}{\tilde{S}+2 \tilde{\lambda}_{j}^{2}}$, we obtain

$$
\begin{aligned}
& t \tilde{S}^{2}\left\{\tilde{S}-2 n-\left[5-t-\left(1-c_{1}\right) u\right] \frac{\tilde{S}}{n}\right\}+\left\{5-\left(2-c_{1}\right) u+\frac{\beta}{3}\right\} A \\
& -\left\{6+\left(1+2 c_{1}\right) u-\frac{2 \beta}{3}\right\} B-\left[2-\left(t-c_{1}\right) u\right] \tilde{S} f \\
\geq & \sum_{j}\left(f_{3}\right)_{j}^{2}\left\{\frac{4}{27} \cdot \frac{\beta}{\tilde{S}+2 \tilde{\lambda}_{j}^{2}}+\frac{4 \tilde{\lambda}_{j}^{2}}{9 t \tilde{S}^{2}}-\frac{u\left(c_{0}+1\right)}{3 \tilde{S}}\right\}+g_{2}+g_{4} \\
\geq & \frac{1}{9 \tilde{S}} \sum_{j}\left(f_{3}\right)_{j}^{2}\left\{8 \sqrt{\frac{\beta}{6 t}}-\frac{2}{t}-3 u\left(c_{0}+1\right)\right\}+g_{2}+g_{4} .
\end{aligned}
$$

Taking $u=\frac{13}{3}$ and $\beta=14$ in the inequality above, we get $14=\frac{\left\{\frac{13}{3}\left(c_{0}+1\right)-1\right\}^{2}}{\frac{13}{3} c_{0}}-t$ and

$$
\begin{aligned}
& t \tilde{S}^{2}\left\{\tilde{S}-2 n-\left[5-t-\frac{13}{3}\left(1-c_{1}\right)\right] \frac{\tilde{S}}{n}\right\}+\left\{5-\frac{13}{3}\left(2-c_{1}\right)+\frac{14}{3}\right\} A \\
& \quad-\left\{6+\frac{13}{3}\left(1+2 c_{1}\right)-\frac{28}{3}\right\} B-\left\{2-\frac{13}{3}\left(t-c_{1}\right)\right\} \tilde{S} f \\
& \geq \frac{1}{\tilde{S}} \sum_{j}\left(\sum_{i} \lambda_{i}^{2} h_{i i j}\right)^{2}\left\{8 \sqrt{\frac{7}{3 t}}-\frac{2}{t}-13\left(c_{0}+1\right)\right\}+g_{2}+g_{4} .
\end{aligned}
$$

Set

$$
\frac{13}{3} \theta_{1}(t)=8 \sqrt{\frac{7}{3 t}}-\frac{2}{t}-13\left(c_{0}+1\right)
$$


Because $\frac{d \theta_{1}(t)}{d t}<0$ for $\frac{1}{9}<t<\frac{1}{4}, \theta_{1}(t) \geq \theta_{1}\left(\frac{1}{4}\right) \geq-\frac{3}{5}$. It follows from (3.5) and $t<\frac{1}{4}$ that

$$
\begin{aligned}
& t \tilde{S}^{2}\left\{\tilde{S}-2 n+\left(t-\frac{13}{3} c_{1}-\frac{2}{3}\right) \frac{\tilde{S}}{n}\right\} \\
\geq & -\left(1+\frac{13}{3} c_{1}\right) A+\left(1+\frac{26}{3} c_{1}\right) B+\left(2+\frac{13}{3} c_{1}-4 t \frac{13}{10}\left(1+\frac{\alpha}{3}\right)\right) \tilde{S} f+g_{2}+g_{4} \\
\geq & -\left(1+\frac{13}{3} c_{1}\right) A+\left(1+\frac{26}{3} c_{1}\right) B+\left(2+\frac{13}{3} c_{1}-\frac{13}{10}\left(1+\frac{\alpha}{3}\right)\right) \tilde{S} f+g_{2}+g_{4} \\
= & -\left(1+\frac{13}{3} c_{1}\right) A+\left(1+\frac{26}{3} c_{1}\right) B+\left(\frac{7}{10}+\frac{13}{3}\left(c_{1}-\frac{\alpha}{10}\right)\right) \tilde{S} f+g_{2}+g_{4} .
\end{aligned}
$$

Taking $c_{1}=\frac{\alpha}{10}$ in above inequality, we see $c_{1} \in\left[0, \frac{1}{10}\right]$ from the definition of $\alpha$. By (3.3), (3.4) and $A+2 B=\frac{1}{3} \sum_{i, j, k}\left(\lambda_{i}+\lambda_{j}+\lambda_{k}\right)^{2} h_{i j k}^{2} \geq 0$, we have

$$
\begin{aligned}
& t \tilde{S}^{2}\left\{\tilde{S}-2 n+\left(t-\frac{2}{3}-\frac{13}{30} \alpha\right) \frac{\tilde{S}}{n}\right\} \\
\geq & -\left(1+\frac{13}{30} \alpha\right) A+\left(1+\frac{26}{30} \alpha\right) B+\frac{7}{10} \tilde{S} f+g_{2}+g_{4} \\
= & -\left(1+\frac{13}{30} \alpha \cdot \frac{4}{3}\right)(A-B)+\frac{13}{30} \alpha \cdot \frac{1}{3}(A+2 B)+\frac{7}{10} \tilde{S} f+g_{2}+g_{4} \\
\geq & -\left(1+\frac{26}{45} \alpha\right)(A-B)+\frac{7}{10} \tilde{S} f+g_{2}+g_{4} \\
\geq & -\left(1+\frac{26}{45} \alpha\right) \frac{1-\alpha}{3}\left(\tilde{\lambda}_{1}-\tilde{\lambda}_{2}\right)^{2} t \tilde{S}^{2}+\frac{7}{10} \tilde{S} \frac{\left(\tilde{\lambda}_{1}-\tilde{\lambda}_{2}\right)^{2}}{\tilde{\lambda}_{1}^{2}+\tilde{\lambda}_{2}^{2}}\left(\tilde{\lambda}_{1} \tilde{\lambda}_{2}+\frac{\tilde{S}}{n}\right)^{2}+g_{2}+g_{4} .
\end{aligned}
$$

By $\sum_{i} \tilde{\lambda}_{i}=0$ and $\sum_{i} \tilde{\lambda}_{i}^{2}=\tilde{S}$, we get $\tilde{\lambda}_{1} \tilde{\lambda}_{2} \leq 0$ and $\frac{\left(\tilde{\lambda}_{1}-\tilde{\lambda}_{2}\right)^{2}}{\tilde{\lambda}_{1}^{2}+\tilde{\lambda}_{2}^{2}} \geq 1$. Since $-(1+$ $\left.\frac{26}{45} \alpha\right)(1-\alpha)=\frac{26}{45}(\alpha-1)\left(\alpha+\frac{45}{26}\right) \geq-1(0 \leq \alpha \leq 1)$, we have

$$
\begin{aligned}
& t \tilde{S}^{2}\left\{\tilde{S}-2 n+\left(t-\frac{2}{3}-\frac{13}{30} \alpha\right) \frac{\tilde{S}}{n}\right\} \\
\geq & -\frac{1}{3}\left(\tilde{\lambda}_{1}^{2}+\tilde{\lambda}_{2}^{2}-2 \tilde{\lambda}_{1} \tilde{\lambda}_{2}\right) t \tilde{S}^{2}+\frac{7}{10} \tilde{S}\left(\tilde{\lambda}_{1} \tilde{\lambda}_{2}+\frac{\tilde{S}}{n}\right)^{2}+g_{2}+g_{4} \\
\geq & -\frac{1}{3}(\tilde{S}+2 x \tilde{S}) t \tilde{S}^{2}+\frac{2}{3} \tilde{S}^{3}\left(\frac{1}{n}-x\right)^{2}+g_{2}+g_{4} \quad\left(x:=-\frac{\tilde{\lambda}_{1} \tilde{\lambda}_{2}}{\tilde{S}}\right) \\
= & -\frac{1}{3} t \tilde{S}^{3}\left(1+\frac{2}{n}\right)+\frac{2}{3} \tilde{S}^{3}\left[\left(x-\frac{1}{n}\right)^{2}-\left(x-\frac{1}{n}\right) t\right]+g_{2}+g_{4} \\
\geq & -\frac{1}{3} t \tilde{S}^{3}\left(1+\frac{2}{n}\right)-\frac{1}{6} t^{2} \tilde{S}^{3}+g_{2}+g_{4} .
\end{aligned}
$$

On the other hand,

$$
t \tilde{S}^{2}\left\{\tilde{S}-2 n+\left(t-\frac{2}{3}-\frac{13}{30} \alpha\right) \frac{\tilde{S}}{n}\right\} \leq t \tilde{S}^{2}\left\{\tilde{S}-2 n+\left(t-\frac{2}{3}\right) \frac{\tilde{S}}{n}\right\} .
$$

This implies

$$
t \tilde{S}^{2}\left\{\tilde{S}-2 n+\left(t-\frac{2}{3}\right) \frac{\tilde{S}}{n}+\frac{1}{3}\left(1+\frac{2}{n}\right) \tilde{S}+\frac{1}{6} t \tilde{S}\right\} \geq g_{2}+g_{4} .
$$


It follows from (3.1) that

$$
n=\tilde{S}(1-t)+\frac{n H\left[H \tilde{S}-\left(f_{3}-H S\right)\right]}{\tilde{S}} .
$$

Hence

$$
\begin{aligned}
t & \tilde{S}^{2}\left\{\tilde{S}\left[1-2(1-t)+\left(t-\frac{2}{3}\right) \frac{1}{n}+\frac{1}{3}\left(1+\frac{2}{n}\right)+\frac{1}{6} t\right]\right. \\
& \left.-2 \frac{n H\left[H \tilde{S}-\left(f_{3}-H S\right)\right]}{\tilde{S}}\right\} \\
=t & \tilde{S}^{2}\left\{\tilde{S}\left[\left(\frac{13}{6}+\frac{1}{n}\right) t-\frac{2}{3}\right]-2 \frac{n H\left[H \tilde{S}-\left(f_{3}-H S\right)\right]}{\tilde{S}}\right\} \geq g_{2}+g_{4},
\end{aligned}
$$

i.e.,

$$
\left(\frac{13}{6}+\frac{1}{n}\right) t-\frac{2}{3} \geq \frac{g_{2}+g_{4}}{t \tilde{S}^{3}}+2 \frac{n H\left[H \tilde{S}-\left(f_{3}-H S\right)\right]}{\tilde{S}^{2}} .
$$

Using $\left(\frac{13}{6}+\frac{1}{n}\right) t-\frac{2}{3} \leq\left(\frac{13}{6}+\frac{1}{4}\right) t-\frac{2}{3}=\frac{29}{12}\left(t-\frac{8}{29}\right)$, we obtain

$$
t \geq \frac{8}{29}+\frac{12}{29}\left\{\frac{g_{2}+g_{4}}{t \tilde{S}^{3}}+2 \frac{n H\left[H \tilde{S}-\left(f_{3}-H S\right)\right]}{\tilde{S}^{2}}\right\}
$$

Therefore, there exists a positive constant $C_{1}(n)$ depending only on $n$ such that if $|H|<C_{1}(n)$, then

$$
\left|\frac{g_{2}+g_{4}}{t \tilde{S}^{3}}+2 \frac{n H\left[H \tilde{S}-\left(f_{3}-H S\right)\right]}{\tilde{S}^{2}}\right| \leq \frac{1}{16}
$$

and $t \geq \frac{1}{4}$. This contradicts with $t \in\left[\frac{1}{9}, \frac{1}{4}\right)$. So we have $t \geq \frac{1}{4}$ when $|H|<C_{1}(n)$.

Secondly we prove that $t \geq \frac{3}{10}+\varepsilon(n)$, where

$$
\varepsilon(n)= \begin{cases}0.0026, & \mathrm{n}>6 \\ 0.0008, & \mathrm{n}=6 \\ 0.0016, & \mathrm{n}=5 \\ 0.0023, & \mathrm{n}=4\end{cases}
$$

Suppose that $t \in\left[\frac{1}{4}, \frac{3}{10}+\varepsilon(n)\right]$. In (4.11), take $u=\frac{17}{5}, c_{0}=\frac{6}{17}$ and let

$$
5-2 u+c_{1} u+\frac{\beta}{3}=6+u+2 c_{1} u-\frac{2 \beta}{3} .
$$

This together with the definition of $\beta$ gives $\beta=\frac{54}{5}-t$ and $c_{1}=-\frac{2}{17}-\frac{5}{17} t$.

Thus (4.11) can be written as

$$
\begin{aligned}
& t \tilde{S}^{2}\left\{\tilde{S}-2 n-\left(\frac{6}{5}-2 t\right) \frac{\tilde{S}}{n}\right\} \\
\geq & -\left(\frac{7}{5}-\frac{4 t}{3}\right)(A-B)+\left(\frac{8}{5}-\frac{22 t}{5}\right) \tilde{S} f+\frac{\theta_{2}(t)}{\tilde{S}} \sum_{j}\left(\sum_{i} \lambda_{i}^{2} h_{i i j}\right)^{2}+g_{2}+g_{4},
\end{aligned}
$$


where

$$
\theta_{2}(t)=4 \sqrt{\frac{2(n+2)}{3 n t}\left(\frac{54}{5}-t\right)}-\frac{2(n+2)}{n t}-\frac{69}{5} .
$$

It is easy to see that $\frac{d \theta_{2}(t)}{d t}<0$, for $\frac{1}{4} \leq t \leq 0.3026$. Hence $\theta_{2}(t)$ is decreasing for $t \in\left[\frac{1}{4}, 0.3026\right]$. We consider the following cases.

Case $(\mathbf{a}) n>6$. Since $\theta_{2}(t) \leq \theta_{2}\left(\frac{1}{4}\right)<0$ holds for $n>6$, by (3.5), we have

$$
\begin{aligned}
& t \tilde{S}^{2}\left\{\tilde{S}-2 n-\left(\frac{6}{5}-2 t\right) \frac{\tilde{S}}{n}\right\} \\
\geq & -\left(\frac{7}{5}-\frac{4 t}{3}\right)(A-B)+\left(a_{2}(t)-b_{2}(t) \alpha\right) t \tilde{S} f+g_{2}+g_{4},
\end{aligned}
$$

where

$$
a_{2}(t)=\frac{8-22 t}{5 t}+\frac{1}{3} \theta_{2}(t), b_{2}(t)=-\frac{2}{3} \theta_{2}(t) .
$$

When $a_{2}(0.3026)-b_{2}(0.3026) \alpha>0$, from the monotonicity of $\theta_{2}(t)$ for $t \in$ $\left[\frac{1}{4}, 0.3026\right]$, we see that $a_{2}(t)-b_{2}(t) \alpha \geq a_{2}(0.3026)-b_{2}(0.3026) \alpha>0$. Using (3.3), (3.4) and $x=-\frac{\tilde{\lambda}_{1} \tilde{\lambda}_{2}}{\tilde{S}} \in\left[0, \frac{1}{2}\right]$ as in (5.1), we have

$$
\begin{aligned}
& \tilde{S}-2 n-\left(\frac{6}{5}-2 t\right) \frac{\tilde{S}}{n} \\
\geq & -\left(\frac{7}{5}-\frac{4 t}{3}\right) \frac{1-\alpha}{3}\left(\tilde{\lambda}_{1}-\tilde{\lambda}_{2}\right)^{2}+\left(a_{2}(t)-b_{2}(t) \alpha\right) \frac{\left(\tilde{\lambda}_{1}-\tilde{\lambda}_{2}\right)^{2}}{\tilde{\lambda}_{1}^{2}+\tilde{\lambda}_{2}^{2}}\left(\frac{\tilde{\lambda}_{1} \tilde{\lambda}_{2}}{\tilde{S}}+\frac{1}{n}\right)^{2} \tilde{S} \\
& +\frac{g_{2}+g_{4}}{t \tilde{S}^{2}} \\
\geq & -\left(\frac{7}{15}-\frac{4 t}{9}\right)(1-\alpha)(1+2 x) \tilde{S}+\left(a_{2}(t)-b_{2}(t) \alpha\right)(1+2 x)\left(\frac{1}{n}-x\right)^{2} \tilde{S} \\
& +\frac{g_{2}+g_{4}}{t \tilde{S}^{2}} \\
= & (\rho(x, n)+\delta(x, n) \alpha) \tilde{S}+\frac{g_{2}+g_{4}}{t \tilde{S}^{2}},
\end{aligned}
$$

where

$$
\begin{aligned}
& \rho(x, n)=-\left(\frac{7}{15}-\frac{4 t}{9}\right)(1+2 x)+a_{2}(t)(1+2 x)\left(\frac{1}{n}-x\right)^{2}, \\
& \delta(x, n)=\left(\frac{7}{15}-\frac{4 t}{9}\right)(1+2 x)-b_{2}(t)(1+2 x)\left(\frac{1}{n}-x\right)^{2} .
\end{aligned}
$$

By the monotonicity of $\rho(x, n)$ and $\delta(x, n)$ (see [16]), we get

$$
\begin{gathered}
\delta(x, n) \geq \min \left\{\delta(0, n), \delta\left(\frac{1}{2}, n\right)\right\}>0, \\
\rho(x, n)+\left(\frac{6}{5}-2 t\right) \frac{1}{n} \geq\left.\min \left\{\rho(x, n)+\left(\frac{6}{5}-2 t\right) \frac{1}{n}\right\}\right|_{t=0.3026}=-0.3943 .
\end{gathered}
$$


So

$$
\tilde{S}-2 n \geq-0.3943 \tilde{S}+\frac{g_{2}+g_{4}}{t \tilde{S}^{2}} .
$$

It follows from (5.2) that

$$
t \geq 0.3028+\frac{g_{2}+g_{4}}{2 t \tilde{S}^{3}}+\frac{n H\left[H \tilde{S}-\left(f_{3}-H S\right)\right]}{\tilde{S}^{2}} .
$$

When $a_{2}(0.3026)-b_{2}(0.3026) \alpha \leq 0$, i.e., $\frac{a_{2}(0.3026)}{b_{2}(0.3026)} \leq \alpha \leq 1$, it follows from (3.4), (3.7) and $a_{2}(t)-b_{2}(t) \alpha \geq a_{2}(0.3026)-b_{2}(0.3026) \alpha$ that

$$
\begin{aligned}
& t \tilde{S}^{2}\left\{\tilde{S}-2 n-\left(\frac{6}{5}-2 t\right) \frac{\tilde{S}}{n}\right\} \\
\geq & -\left(\frac{7}{15}-\frac{4 t}{9}\right)(1-\alpha)\left(\tilde{\lambda}_{1}-\tilde{\lambda}_{2}\right)^{2} t \tilde{S}^{2} \\
& +\left(a_{2}(0.3026)-b_{2}(0.3026) \alpha\right)\left[\frac{\left(\tilde{\lambda}_{1}-\tilde{\lambda}_{2}\right)^{2}}{4}-\frac{\tilde{S}}{n}\right] t \tilde{S}^{2}+g_{2}+g_{4} .
\end{aligned}
$$

Noting that $\left(\tilde{\lambda}_{1}-\tilde{\lambda}_{2}\right)^{2} \leq 2 \tilde{S}$, we get

$$
\begin{aligned}
& \tilde{S}-2 n-\left(\frac{6}{5}-2 t\right) \frac{\tilde{S}}{n} \\
\geq & \left\{-2\left(\frac{7}{15}-\frac{4 t}{9}\right)+\frac{n-2}{2 n} a_{2}(0.3026)+\left[2\left(\frac{7}{15}-\frac{4 t}{9}\right)-\frac{n-2}{2 n} b_{2}(0.3026)\right] \alpha\right\} \tilde{S} \\
& +\frac{g_{2}+g_{4}}{t \tilde{S}^{2}} .
\end{aligned}
$$

Since $b_{2}(0.3026)<0.7821$ and the assumption of $t<0.3026$, we have $2\left(\frac{7}{15}-\frac{4 t}{9}\right)-$ $\frac{n-2}{2 n} b_{2}(0.3026)>0$. Using $\alpha \geq \frac{a_{2}(0.3026)}{b_{2}(0.3026)}$, we obtain

$$
\tilde{S}-2 n-\left(\frac{6}{5}-2 t\right) \frac{\tilde{S}}{n} \geq-2\left(\frac{7}{15}-\frac{4 t}{9}\right)\left[1-\frac{a_{2}(0.3026)}{b_{2}(0.3026)}\right] \tilde{S}+\frac{g_{2}+g_{4}}{t \tilde{S}^{2}} .
$$

By a direct computation, one see that $\frac{a_{2}(0.3026)}{b_{2}(0.3026)} \geq 0.6348$. Then

$$
\begin{aligned}
\tilde{S}-2 n-\left(\frac{6}{5}-2 t\right) \frac{\tilde{S}}{n} & \geq-2\left(\frac{7}{15}-\frac{4 t}{9}\right) 0.3652 \tilde{S}+\frac{g_{2}+g_{4}}{t \tilde{S}^{2}} \\
& =-\frac{0.7303}{45} \tilde{S}-\frac{2.9215}{9} n+g_{5},
\end{aligned}
$$

where

$$
g_{5}=\frac{g_{2}+g_{4}}{t \tilde{S}^{2}}+\frac{2.9215 n H\left[H \tilde{S}-\left(f_{3}-H S\right)\right]}{9 \tilde{S}} .
$$

For $t \in\left[\frac{1}{4}, 0.3026\right]$, we have $\left(\frac{6}{5}-2 t\right) \frac{\tilde{S}}{n}>0$. It is seen from (5.4) that

$$
\tilde{S}-2 n>-\frac{0.7303}{45} \tilde{S}-\frac{2.9215}{9} n+g_{5} .
$$


Therefore, using (5.2), we get

$$
t>0.3934+\frac{n H\left[H \tilde{S}-\left(f_{3}-H S\right)\right]}{\tilde{S}^{2}}+\frac{0.5968 g_{5}}{\tilde{S}} .
$$

From (5.3) and (5.5), we see that there is a positive constant $C_{2}(n)(n>6)$ such that if $|H|<C_{2}(n)$, then

$$
\left|\frac{g_{2}+g_{4}}{2 t \tilde{S}^{3}}+\frac{n H\left[H \tilde{S}-\left(f_{3}-H S\right)\right]}{\tilde{S}^{2}}\right|<0.0002,
$$

and

$$
\left|\frac{n H\left[H \tilde{S}-\left(f_{3}-H S\right)\right]}{\tilde{S}^{2}}+\frac{0.5968 g_{5}}{\tilde{S}}\right|<0.0908,
$$

which imply $t>0.3026$. This makes a contradiction. Hence we have $t>0.3026$ when $|H|<C_{2}(n)(n>6)$.

Case $(\mathbf{b}) 4 \leq n \leq 6$. We take $u=\frac{7}{2}, c_{0}=\frac{61-\sqrt{1221}}{70}$ in (4.11), and get

$$
\begin{aligned}
& t \tilde{S}^{2}\left\{\tilde{S}-2 n-\left(\frac{11}{10}-2 t\right) \frac{\tilde{S}}{n}\right\} \\
\geq & -\left(\frac{13}{10}-\frac{4 t}{3}\right)(A-B)+\left(\frac{8}{5}-\frac{9 t}{2}\right) \tilde{S} f+\frac{\theta_{3}(t)}{\tilde{S}} \sum_{j}\left(\sum_{i} \lambda_{i}^{2} h_{i i j}\right)^{2}+g_{2}+g_{4},
\end{aligned}
$$

where

$$
\theta_{3}(t)=4 \sqrt{\frac{2(n+2)}{3 n t}\left(\frac{111}{10}-t\right)}-\frac{2(n+2)}{n t}-\frac{393-3 \sqrt{1221}}{20} .
$$

One can see that $\theta_{3}(t)$ is decreasing for $t \in\left[\frac{1}{4}, 0.3023\right]$ and $\theta_{3}(t) \leq \theta_{3}\left(\frac{1}{4}\right)<0$ holds for $4 \leq n \leq 6$. It follows from (3.5) that

$$
\begin{aligned}
& t \tilde{S}^{2}\left\{\tilde{S}-2 n-\left(\frac{11}{10}-2 t\right) \frac{\tilde{S}}{n}\right\} \\
\geq & -\left(\frac{13}{10}-\frac{4 t}{3}\right)(A-B)+\left(a_{3}(t)-b_{3}(t) \alpha\right) t \tilde{S} f+g_{2}+g_{4}
\end{aligned}
$$

where

$$
a_{3}(t)=\frac{8}{5 t}-\frac{9}{2}+\frac{1}{3} \theta_{3}(t), b_{3}(t)=-\frac{2}{3} \theta_{3}(t) .
$$

For $4 \leq n \leq 6$ and $\frac{1}{4} \leq t \leq 0.3061$, we have $a_{3}(t)>b_{3}(t)>0$. Thus $a_{3}(t)-b_{3}(t) \alpha \geq$ $a_{3}(t)-b_{3}(t)>0$. By (3.3) and (3.4), we repeat the computational procedure in Case(a) for $n \in\{4,5,6\}$, respectively. From the assumption of $t \leq \frac{3}{10}+\varepsilon(n)$, we obtain

$$
\tilde{S}-2 n>-\left(\frac{4}{10}-2 \varepsilon^{\prime}(n)\right) \tilde{S}+\frac{g_{2}+g_{4}}{t \tilde{S}^{2}}
$$


where

$$
\varepsilon^{\prime}(n)= \begin{cases}0.0009, & \mathrm{n}=6 \\ 0.0017, & \mathrm{n}=5 \\ 0.0025, & \mathrm{n}=4\end{cases}
$$

It follows from (5.2) that

$$
t>\frac{3}{10}+\varepsilon^{\prime}(n)+\frac{g_{2}+g_{4}}{2 t \tilde{S}^{3}}+\frac{n H\left[H \tilde{S}-\left(f_{3}-H S\right)\right]}{\tilde{S}^{2}} .
$$

Then there exists a positive constant $C_{2}(n)(\mathrm{n}=4,5,6)$ such that $t>\frac{3}{10}+\varepsilon(n)$ when $|H|<C_{2}(n)$. This is a contradiction. Hence we have $t>\frac{3}{10}+\varepsilon(n)$ for $n=4,5,6$.

It is known in [12] that

$$
\tilde{S}+\frac{n(n-2) H}{\sqrt{n(n-1)}} \sqrt{\tilde{S}}-n-n H^{2} \geq t \tilde{S} .
$$

So

$$
\begin{aligned}
\tilde{S} \geq & n \sigma+\frac{n H^{2}}{2(n-1)} \sigma\left((n-2)^{2} \sigma+2(n-1)\right) \\
& -\frac{n(n-2) H}{2(n-1)} \sigma^{2} \sqrt{(n-2)^{2} H^{2}+\frac{4\left(H^{2}+1\right)(n-1)}{\sigma}} \\
\geq & n \sigma+n H^{2}\left(\frac{n^{2}}{2(n-1)}-1\right)-\frac{n(n-2) H}{n-1} \sqrt{n^{2} H^{2}+4(n-1)},
\end{aligned}
$$

where $\sigma=\frac{1}{1-t}$, and $1<\sigma<2$ for $0<t<\frac{1}{2}$. Then

$$
\begin{aligned}
S-\beta(n, H)> & n H^{2}-\beta(n, H)+n \sigma+n H^{2}\left(\frac{n^{2}}{2(n-1)}-1\right) \\
& -\frac{n(n-2) H}{n-1} \sqrt{n^{2} H^{2}+4(n-1)} \\
= & n H^{2}-n-\frac{n^{3} H^{2}}{2(n-1)}+n \sigma+n H^{2}\left(\frac{n^{2}}{2(n-1)}-1\right) \\
& -\frac{3 n(n-2) H}{2(n-1)} \sqrt{n^{2} H^{2}+4(n-1)} \\
= & n(\sigma-1)-\frac{3 n(n-2) H}{2(n-1)} \sqrt{n^{2} H^{2}+4(n-1)} .
\end{aligned}
$$

If $H \leq \frac{2}{n}$ and $t>\frac{3}{10}+\varepsilon(n)$, we have

$$
S-\beta(n, H)>\frac{n t}{1-t}-\frac{3(n-2) n^{\frac{3}{2}}}{n-1} H>\frac{3 n}{7}+\frac{100 n}{49} \varepsilon(n)-\frac{3(n-2) n^{\frac{3}{2}}}{n-1} H .
$$

Set $C(n)=\min \left\{C_{1}(n), C_{2}(n), \frac{2}{n}, \frac{100(n-1)}{147(n-2) \sqrt{n}} \varepsilon(n)\right\}$ and assume $|H|<C(n)$. We conclude that if $S>\beta(n, H)$, then $S>\beta(n, H)+\frac{3}{7} n$. This completes the proof of our main theorem. 


\section{REFERENCES}

[1] H. Alencar And M. Do CARmo, Hypersurfaces with constant mean curvature in spheres, Proc. Amer. Math. Soc., 120 (1994), pp. 1223-1229.

[2] S. P. Chang, On minimal hypersurfaces with constant scalar curvatures in $S^{4}$, J. Diff. Geom., 37 (1993), pp. 523-534.

[3] S. P. Chang, A closed hypersurface of constant scalar and mean curvatures in $\mathrm{S}^{4}$ is isoparametric, Comm. Anal. Geom., 1 (1993), pp. 71-100.

[4] Q. M. Cheng, Y. J. He, And H. Z. LI, Scalar curvature of hypersurfaces with constant mean curvature in a sphere, Glasgow Math. J., 51 (2009), pp. 413-423.

[5] Q. M. Cheng and S. Ishikawa, A characterization of the Clifford torus, Proc. Amer. Math. Soc., 127 (1999), pp. 819-828.

[6] Q. M. Cheng And H. NAKagawa, Totally umbilic hypersurfaces, Hiroshima Math. J., 20 (1990), pp. $1-10$.

[7] S. Y. Cheng, On the Chern conjecture for minimal hypersurface with constant scalar curvatures in the spheres, Tsing Hua Lectures on Geometry and Analysis, International Press, Cambridge, MA, 1997, pp. 59-78.

[8] S. S. Chern, M. do Carmo, and S. Kobayashi, Minimal submanifolds of a sphere with second fundamental form of constant length, Functional Analysis and Related Fields, SpringerVerlag, Berlin, 1970, pp. 59-75.

[9] Q. Ding AND Y. L. XIN, On Chern's problem for rigidity of minimal hypersurfaces in the spheres, Advances in Math., 227 (2011), pp. 131-145.

[10] H. B. LaWson JR., Local rigidity theorems for minimal hypersurfaces, Ann. of Math., 89 (1969), pp. $167-179$.

[11] A. M. Li And J. M. LI, An intrinsic rigidity theorem for minimal submanifolds in a sphere, Arch. Math., 58 (1992), pp. 582-594.

[12] M. OKumura, Hypersurfaces and a pinching problem on the second fundamental tensor, Amer. J. Math., 96 (1974), pp. 207-213.

[13] C. K. Peng And C. L. Terng, Minimal hypersurfaces of spheres with constant scalar curvature, Seminar on Minimal Submanifolds, Annals of Mathematics Studies, Vol. 103 (Princeton Univ. Press, Princedon, 1983), pp. 179-198.

[14] C. K. Peng and C. L. Terng, The scalar curvature of minimal hypersurfaces in sphere, Math. Ann., 266 (1983), pp. 105-113.

[15] J. Simons, Minimal varieties in Riemannian manifolds, Ann. of Math., 88 (1968), pp. 62-105.

[16] Y. J. SUH AND H. Y. YANG, The scalar curvature of minimal hypersurfaces in a unit sphere, Commun. Contemp. Math., 9 (2007), pp. 183-200.

[17] S. M. Wei AND H. W. XU, Scalar curvature of minimal hypersurfaces in a sphere, Math. Res. Lett., 14 (2007), pp. 423-432.

[18] H. W. Xu, Pinching theorems, global pinching theorems and eigenvalues for Riemannian submanifolds, Ph.D. dissertation, Fudan University, 1990.

[19] H. W. XU, A rigidity theorem for submanifolds with parallel mean curvature in a sphere, Arch. Math., 61 (1993), pp. 489-496.

[20] H. W. Xu, A gap of scalar curvature for higher dimensional hypersurfaces with constant mean curvature, Appl. Math. J. Chinese Univ. Ser. A, 8 (1993), pp. 410-419.

[21] H. W. XU AND Z. Y. XU, The second pinching theorem for hypersurfaces with constant mean curvature in a sphere, arXiv: math.DG/1012.2173.

[22] H. W. Xu And E. T. ZhaO, A characterization of Clifford hypersurface, preprint, 2008.

[23] Y. W. Xu And Z. Y. Xu, On rigidity of Clifford torus in a unit sphere, Appl. Math. J. Chinese Univ. Ser. B, 26 (2011), pp. 121-126.

[24] H. C. Yang AND Q. M. Cheng, An estimate of the pinching constant of minimal hypersurfaces with constant scalar curvature in the unit sphere, Manuscipta Math., 84 (1994), pp. 89-100.

[25] H. C. Yang and Q. M. Cheng, Chern's conjecture on minimal hypersurfaces, Math. Z., 227 (1998), pp. 377-390. 
